\title{
SPATIAL EQUITY AND HIGH-SPEED RAIL SYSTEMS
}

\author{
L. BIGGIERO, F. PAGLIARA, A. PATRONE \& F. PERUGGINI \\ Department of Civil, Architectural and Environmental Engineering, University of Naples Federico II, Italy
}

\begin{abstract}
In the literature by spatial accessibility it is meant a measure of spatial equity represented by the ease of travelling from an origin to a destination with a given transport mode. Recent investments in highspeed rail (HSR) systems have brought an increase in accessibility as well as equity issues, which will be discussed in this contribution.

Indeed equity impacts, due to the extension of the HSR network in Italy, are here evaluated through the results of a joint revealed preference/stated preference (RP/SP) survey. In the first part of the questionnaire submitted to Italian travellers, i.e. the RP exercise, socio-economic data about the users together with information concerning their trip have been collected. In the second part of the questionnaire, the SP exercise has been employed. Specifically, nine hypothetical scenarios have been submitted to respondents with the objective of understanding what was the transport mode chosen, within a given context, and to see whether HSR was the preferred alternative (or it was an element of spatial exclusion).

The main policy implications of this study are that investors in HS should take into account not only the economic benefits brought by them, but also the spatial imbalance brought by these systems.

Keywords: spatial equity, accessibility; high-speed rail investments.
\end{abstract}

\section{INTRODUCTION}

Several papers have been proposed in the literature to deal with the question of spatial equity and transportation systems, especially for road transport [1]. However, not many contributions have been provided on the link with high-speed rail (HSR).

Spatial equity or spatial accessibility can be considered as a measure of the ease of travelling from an origin to a given destination via a given mode or set of transport modes.

Urban areas can benefit from improvements in accessibility when a new HSR line is built [2]. These improvements can foster locational advantages and increase the attractiveness of the cities served. However, HSR systems extensions may increase spatial imbalance, leading to more polarized patterns of spatial development. Equal rights of access to different services have been the subject of many researchers for years and several theories have been proposed by scientists.

In the paper by Thomopoulos et al. [3] 'spatial equity refers to the geographical location of an individual, group or region affected by a transport infrastructure project'. The authors develop a framework offering an additional support tool to decision makers for differentiating choices based on their views on given equity principles and equity types.

The achievement of equity in the distribution of public resources is very important for planners. Equitable distribution helps locating facilities and services so that as many different spatially defined social groups can have access. Tale [4] proposes a method according to which planners can generate and evaluate 'equity maps' of resource distribution, through which they can explore the spatial relationships between public facilities and socio-economic characteristics.

This paper is part of the proceedings of the 22nd International Conference on Urban Transport and the Environment (Urban Transport 2016)

www.witconferences.com 
In the paper by Monzón et al. [5], for the case study of Spain, it is shown that an increase in speed from $220 \mathrm{~km} / \mathrm{h}$ to $300 \mathrm{~km} / \mathrm{h}$ in a given corridor brings significant negative impacts on spatial equity between locations with and without an HSR service. They also propose an evaluation methodology for HSR projects following a twofold approach, i.e. addressing issues of both efficiency and equity. The procedure uses spatial impact analysis techniques and is based on the computation of accessibility indicators. Efficiency impacts are evaluated in terms of increased accessibility resulting from the HSR project, with a focus on major urban areas; and spatial equity implications are derived from changes in the distribution of accessibility values among these urban agglomerations [2]. In the authors' opinion, the problem of accessibility should be treated taking also into account the reliability of modes of transport. Indeed transport reliability is typically approached in literature by a management point of view [6,7], but it should be taken into account in the computation of accessibility indicators.

In the paper by Church et al. [8] seven categories of social exclusion related to transport have been proposed. The one related to geographical exclusion is the closer to the concept of spatial equity since it is conceived as the exclusion preventing people from accessing transport services, especially those living in rural areas or on peripheral urban estates.

The results of a revealed preference (RP) survey have shown that for those who have not chosen HSR, the main reason is the geographical exclusion. It follows the economic exclusion, i.e. the cost of the HSR ticket. The objective of this manuscript is to describe a methodology for evaluating the equity impacts on population due to an extension of the HSR network in Italy.

This paper is organized as follows. In Section 2 the case study of Italy will be presented together with the description of the survey. In Section 3 results are presented. In Section 4 some inferences on the relationship between HSR and equity issues are reported. In Section 5 conclusions and further perspectives are described.

\section{THE CASE STUDY}

The first HS line in Europe, designed at the beginning of the 1960s, was the Direttissima Rome-Florence. It was opened in 1981 and it represents the first example of HS rail link in Italy. Due to political and building (orographic) problems which delayed the HS construction, in 2000, Italy had $248 \mathrm{~km}$ of HSR line, i.e. about half of those of Germany and Spain and even 1/5 of that of France. In 2006 there were $562 \mathrm{~km}$ of new lines with the opening of the Rome-Naples (to Gricignano) and of the Turin-Novara HS rail sections. However, in the same period, Spain increased its HSR kilometres from 470 to 1225 [9]. The development of the high-speed/high-capacity (HS/HC) project in Italy is still a 'work in progress'. Apart from the already operating sections other lines will be inaugurated in the coming years. This project has been very expensive; the cost of the section Turin-Milan-Naples has been around 32 billion Euros and it has represented the biggest investment in infrastructures in Italy after the 'motorway age'. The key objectives for the construction that is currently underway are to raise the Italian rail network to the best European standards and to improve its capacity. After the completion of the HSR system there will be a reduction in travel time between the major cities connected in the order of 40-50\%.

The national Italian network and operations are all owned by Ferrovie dello Stato (State Railway) Holdings, a fully government-owned company. It has three key operating subsidiaries: Trenitalia operates all freight and passenger trains, including the high-speed trains; RFI (Rete Ferroviaria Italiana) manages the infrastructure; and TAV (Treno Alta Velocità SpA) is responsible for the planning and construction of the new HS infrastructure [10]. 
Since 2012 a new private company, named Nuovo Treno Viaggiatori (NTV), is competing with Trenitalia on the same HSR network. This represents a unique case in the world since two operators, one public and the other private, compete on the same HSR network.

\section{THE SURVEY}

A survey has been carried out, interviewing Italians with the objective of studying the impacts on population in terms of spatial equity after a possible extension of HSR network. The first part of the questionnaire consists of a RP exercise collecting socio-economic data about the users together with information concerning their trip (i.e. origin/destination, transport mode chosen, travel time and cost). In the second part of the questionnaire, a stated preference (SP) exercise has been defined. Specifically, 9 hypothetical scenarios have been submitted to the respondents with the objective of understanding what was the transport mode chosen within a given context and to see whether HSR was the preferred alternative or it was an element of spatial exclusion. Considering a fractional factorial design, each alternative has been represented by four variables, two of them varying between two levels and the others among three levels. These are access/egress travel time and cost to/from the departure/arrival station; total travel cost; HSR travel cost; and travel cost of the main chosen transport mode. The choice of the three levels for the variables access/egress travel time and cost aims at catching possible effects more or less than linear that is not appreciable by a two levels game (see Table 1).

The questionnaire has been placed on the Google Drive platform and it has been submitted to Italian travellers having at most 5 five different transport mode alternatives, i.e. car, bus, airplane, intercity regional train and HSR. The questionnaire was available to respondents between March and May 2015.

Table 1: SP variables and their levels.

\begin{tabular}{ccccc}
\hline & \multicolumn{3}{c}{ HSR } & Other RP modes \\
\hline \multirow{4}{*}{ Levels } & Access/egress time & Access/egress cost & Ticket cost & Travel cost \\
& $(\%)$ & $(\%)$ & $(\%)$ & $(\%)$ \\
& -20 & -20 & -20 & +20 \\
& -50 & -50 & 0 & 0 \\
\hline
\end{tabular}

Table 2: The SP scenarios.

\begin{tabular}{|c|c|c|c|c|}
\hline & & HSR & & RP chosen mode \\
\hline $\begin{array}{l}\text { Scenario } \\
\text { no. }\end{array}$ & $\begin{array}{c}\text { Access/egress time } \\
(\%)\end{array}$ & $\begin{array}{c}\text { Access/egress cost } \\
(\%)\end{array}$ & $\begin{array}{c}\text { Ticket cost } \\
(\%)\end{array}$ & $\begin{array}{c}\text { Travel cost } \\
(\%)\end{array}$ \\
\hline 1 & -20 & -50 & 0 & +20 \\
\hline 2 & -20 & 0 & -20 & +20 \\
\hline 3 & -50 & 0 & 0 & +20 \\
\hline 4 & 0 & 50 & 0 & +20 \\
\hline 5 & -20 & -20 & 0 & 0 \\
\hline 6 & 0 & -20 & -20 & +20 \\
\hline 7 & -50 & -50 & -20 & 0 \\
\hline 8 & -50 & -20 & 0 & +20 \\
\hline 9 & 0 & 0 & 0 & 0 \\
\hline
\end{tabular}


To the users not actually choosing HSR, some hypothetical scenarios have been proposed. For each scenario, the user is asked about his changing transport mode in favour of HSR. By applying the fractional factorial design, the number of scenarios proposed to each respondent is 9 instead of 36, relative to the full design. The last scenario corresponds to the actual one and it can be seen as a control scenario useful to compare SP and RP choices. In Table 2 all the scenarios are reported.

Scenario no. 7 represents the most advantageous since the levels of the three variables concerning the HSR alternative assume their minimum value in terms of percentages, although the level of the cost variable of the other transport modes is equal to 0 .

In scenario no. 1 the increase of accessibility is assumed together with a decrease of the performances of the actual transport mode.

The total number of questionnaires collected was 810 . Due to the particular sampling procedure, an adjustment was necessary in order to reduce the sample bias matching the actual distribution of the Italian population (from the mobility census data) in terms of gender and age percentages. Other matching could not be carried out because of the fewer social and economic information contained in the mobility data vs census data.

\section{RESULTS}

\subsection{RP survey}

Almost all the interviewers were Italian, thus RP survey showed some typical aspect of Italian travellers. Men travel more than women $(55.6 \%$ vs $44.4 \%)$ and few are the elder people travelling $(12.9 \%)$ while the middle-age class is the more present over the transport network. Probably due to the sampling method, the sample level of education was high if compared to Italian census data. Indeed 50\% revealed a high school degree as more than $40 \%$ has got a university degree (bachelor, master or doctorate). Thus almost $50 \%$ are employed and about $25 \%$ are freelancers. This can be the reason why only $10 \%$ declared a low family income, about $35 \%$ medium, $39 \%$ medium-high and more than $16 \%$ high.

Among all available modes, the train is the most chosen one with the prevalence of HSR (36.8\%) preferred almost as the car (37.1\%). Marginal percentages are relative to intercity bus $(6.5 \%)$ and plane $(8.7 \%)$.

HRS and car are the preferred modes for daily round trips. This indicates that the decrease of travel times due to HSR allows return in the same day, while the lack of available train or plane or bus runs can justify the choice of car. As a matter of fact, plane is the less chosen mode for daily round trip.

The above statement relative to the choice of HSR is confirmed by the reasons which induced such a choice. Most of the interviewers felt the travel time saving (68.6\%) as the real motivation to prefer HSR, followed by comfort (15\%) and cost $(9.4 \%)$. The latter can be ascribed to the large discounts offered by operators to people who buy ticket in advance. Probably due to cultural factors, safety and environment impact has not be considered as reasons to choose HSR. The appreciable effect of the accessibility to the station (6.7\%) introduces the problem of the exclusion to HSR. As is the case for HSR user, people may not take the HS train due to the lack of transport supply to and from stations.

Mode choice seems not to be different between genders. Women seem to reveal a little more preference for public transport and it can be due to the perception of security given by guarded places such as train station and airport. 
Young people prefer public transport while middle aged and elder people divide themselves mainly among car and HSR. The behaviour of young people is to attribute to the survey methodology which sampled a large percentage of university students. Indeed the distribution of the chosen mode over the occupation reveals that students and employees prefer public transport; on the contrary, the freelancers and the others like better speedy and/ or elastic modes such as HSR or car.

\subsection{SP survey}

The SP survey was conducted on the users who don't choose HSR to better investigate the inference of level of service (LoS) variables on the choice of HSR with the objective of highlighting equity issue.

Concerning the variable access/egress travel time, in Fig. 1 it is possible to notice a lessthan-linear effect of users' choice. Moreover, the LoS referred to $-20 \%$ and $-50 \%$ is almost equal and respectively $63 \%$ and $64 \%$. This means that a threshold value can be defined, beyond which there are not evident variations of users' choice. It follows that a decrease of only $-20 \%$ would bring a positive response from HSR users both actual and potential.

Different is the case of the access/egress travel cost for which a more-than-linear trend is evident. In this case, the threshold effect is observed only at $-50 \%$, where the HSR choice increases to $72 \%$.

A decrease of only $20 \%$ of the access/egress travel time matches a positive response of $63 \%$, compared to $55 \%$ in the case of the access/egress travel cost.

The top two graphs of Fig. 1 show, then, that the user is strongly affected by the geographic/economic exclusion factors.

On the other hand, by considering the ticket cost, a minimum reduction of $20 \%$ is sufficient to get an increase of HSR users, assuming that the ticket cost is the main trip cost. This assumption can be supported by comparing the ticket cost variable with access/egress travel cost variable: a small decrease of the HSR ticket cost (20\%) shows an yes percentage of $70 \%$
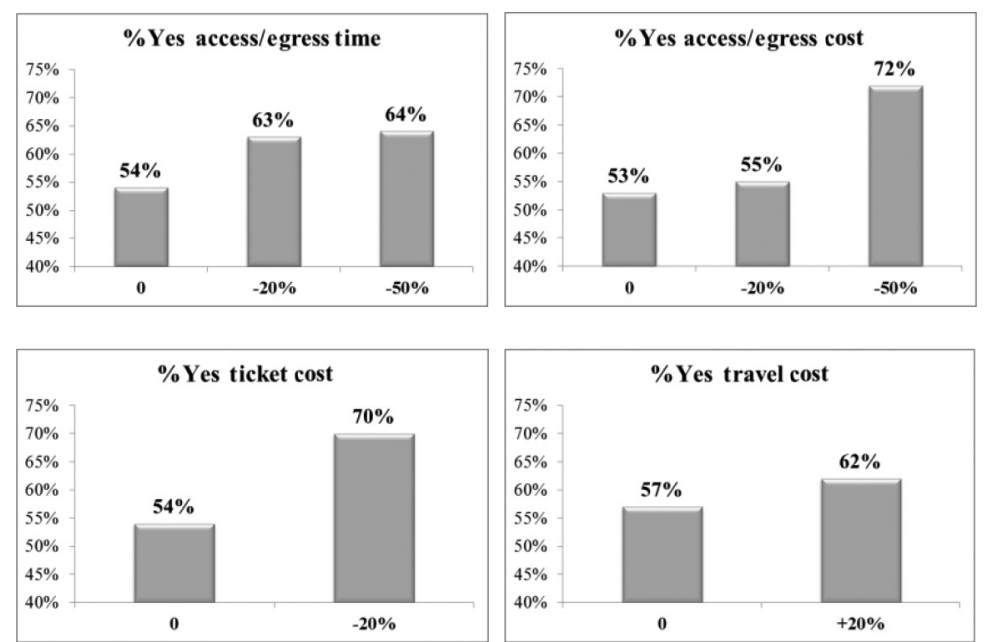

Figure 1: Percentages of other modes users who change to HSR (\% Yes) depending upon different variables. 
whereas a decrease of $50 \%$ of the access/egress travel cost is necessary to obtain the same yes-choice percentage $(72 \%)$.

The last concern on this figure relates the travel cost variable with which it is possible to study the effects on the HSR choice rate of possible increases of travel costs on the non-HSR transport modes. The results show that an increase of $20 \%$ of cost does not greatly influence a transport shift in favour of HSR. This induces to suppose that pull policies have better effects w.r.t. push ones. Probably travellers see the convenience in changing their mode choice due to a pull policy of HSR cost reduction. On the contrary they feel push policies as punishments and they don't change their actual choice since they don't feel HSR as an alternative for their trip.

Since the private transport (car) is prevailing w.r.t. other transport modes, a more deep analysis is needed to understand whether the effects noticed above are the results of car users' choices or if they can be extended to other modes, becoming general effects. In Figs 2 and 3 the same threshold values discussed before can be noted. The only difference is that the yes percentages of the public transport variables are a bit higher than those of car, especially for the ticket cost variable and access/egress cost variable. This is probably due to a higher sensitivity of public transport users towards an out-of-pocket cost decrease. A further confirmation comes from the ticket cost variable for the level ' $-20 \%$ '. Between car users and public transport users there is a difference of $7 \%$ while changing to HSR ( $67 \%$ for car vs $74 \%$ for public transport), probably due to the different perception of the ticket cost of public transport users (for whom a possible decrease of the HSR ticket cost is naturally more perceived), compared with car users (not using to buy a ticket).

An analysis of some scenarios relative to SP survey can better underline combined effects of some variables. In particular, a comparison among scenarios 1, 7 and 9 of Table 2 is analysed. Scenario 9 represents the base scenario referring to actual mode performances instead of scenario 7 which presents the best combination of all variables (in brackets the threshold value discussed before is indicated). Scenario 1 highlights the effects of the best improvement of accessibility to stations (exclusion). Indeed as before stated, the increase of actual mode travel costs does not show relevant effects on mode choice.

Figure 4 shows the very important role of accessibility, i.e. spatial exclusion, in the choice of HSR for intercity trips. The yes percentage for scenario 1 results almost in double $(+35 \%)$ than in the actual situation - increase of another $9 \%$ if a reduction of HSR ticket occurs.
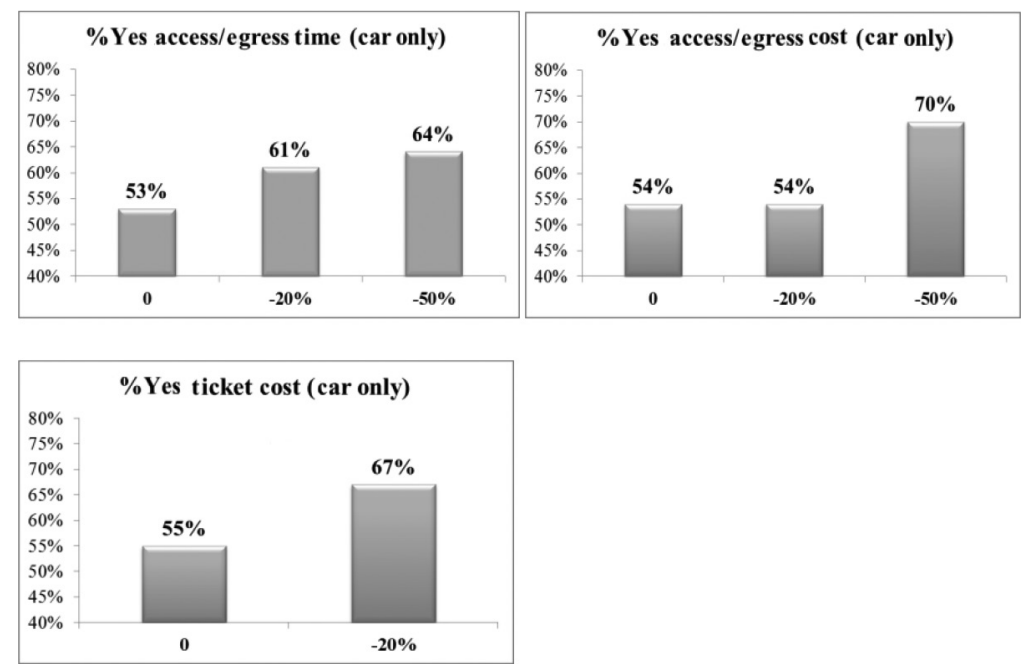

Figure 2: Yes percentages of access/egress travel time, access/egress travel cost and ticket cost related to actual car users. 

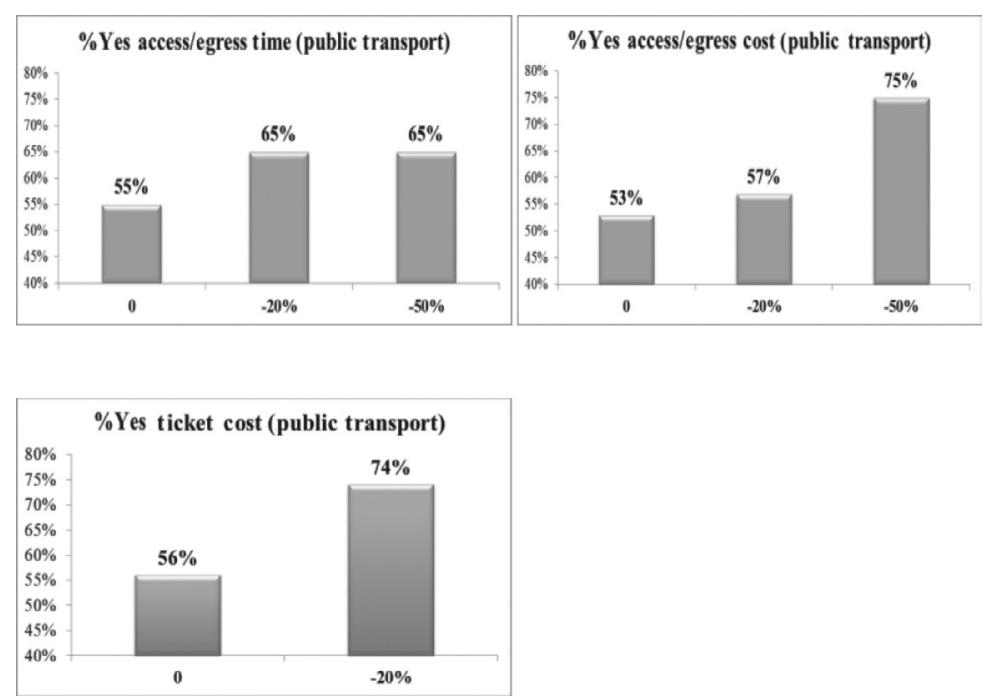

Figure 3: Yes percentages of access/egress travel time, access/egress travel cost and ticket cost related to public transport modes.

Therefore it can be argued how a policy reducing spatial exclusion by reducing time and cost to and from HSR stations has larger effects on travel demand than any other policy related to HSR ticket fares.

Since the lack of accessibility to transport can also be seen as a reason for economic/social exclusion, the sensitivity of mode change to HSR has to be investigated referring to both individual and household income. In Fig. 5 variations in mode choice can be seen w.r.t individual income (Fig. 5a and b) and household income (Fig. 5c and d). The income classes considered are the following three: low, low-medium, and medium-high. As above stated, the effect of decreasing access/egress time is relevant but it seems not to be significantly different among income classes both for individual and household. Indeed concerning the reduction of access/egress travel time of the threshold value of $-20 \%$, low and medium-high income classes reveal to be more elastic and increase their HSR share more than $10 \%$. The improvement of access/egress conditions balances the choice among incomes which become almost equal to 63\% (Fig. 5b).

A similar behaviour can be noticed for the increasing of HSR choice due to the reduction of access/egress costs to the threshold value of $-50 \%$ over the three income classes. Also in this case, the increase is of 10 percentage points on average leading the HSR share to about $71 \%$.

The cut of the HSR ticket fare seems to be more felt as individual income decreases. In Fig. 6 HSR shares start by almost equal percentage values and achieve a difference of $10 \%$

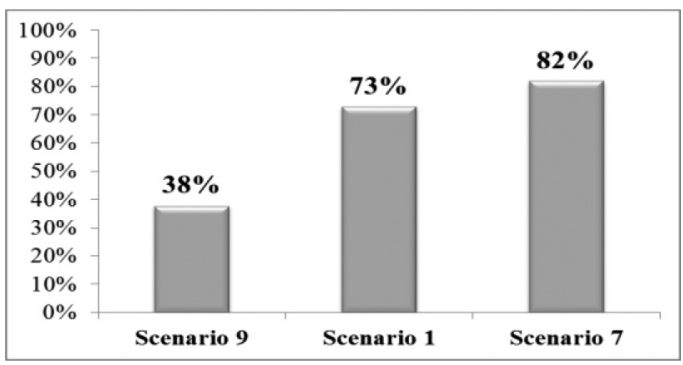

Figure 4: Yes percentages of scenario 9 vs scenarios 1 and 7. 
(a)

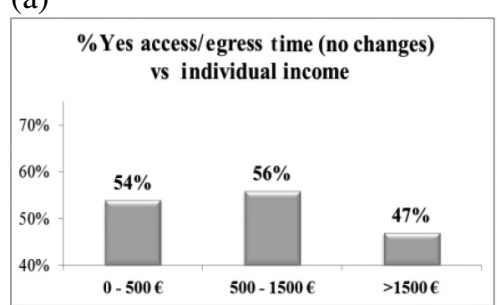

(b)

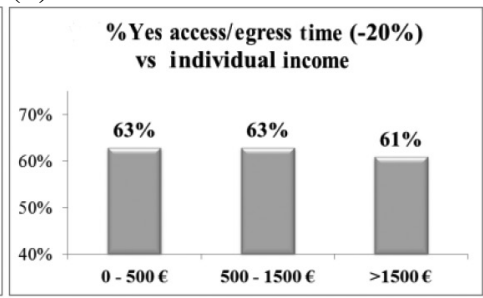

(c)

(d)
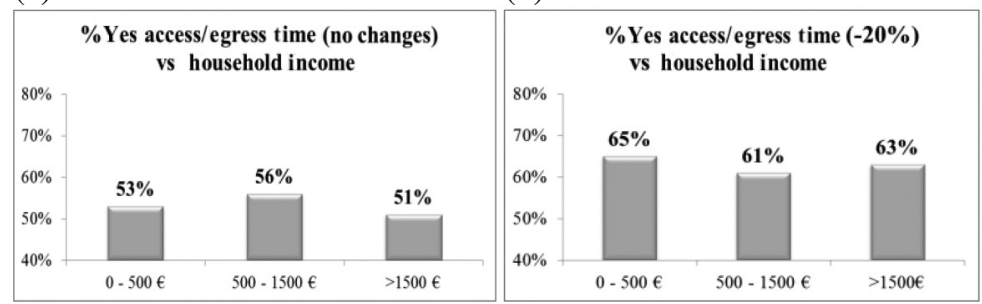

Figure 5: Yes percentages of access/egress travel time (actual situation and level '-20\%') vs individual and household income.

from low to medium-high income when the ticket cut is proposed. It is worth noting that this difference vanishes if household income is considered instead of individual one. This can be due to the high percentage of students in the sample.

Finally the increasing of travel cost relative to actual chosen modes does not show any difference among income.

\section{CONCLUSIONS AND FURTHER DEVELOPMENTS}

The qualitative analysis described in this paper has well shown that in Italy the problem of economic/geographic exclusion does exist and it is seriously perceived by travellers. Indeed a high sensitivity is registered for the access/egress travel costs and also for the HSR

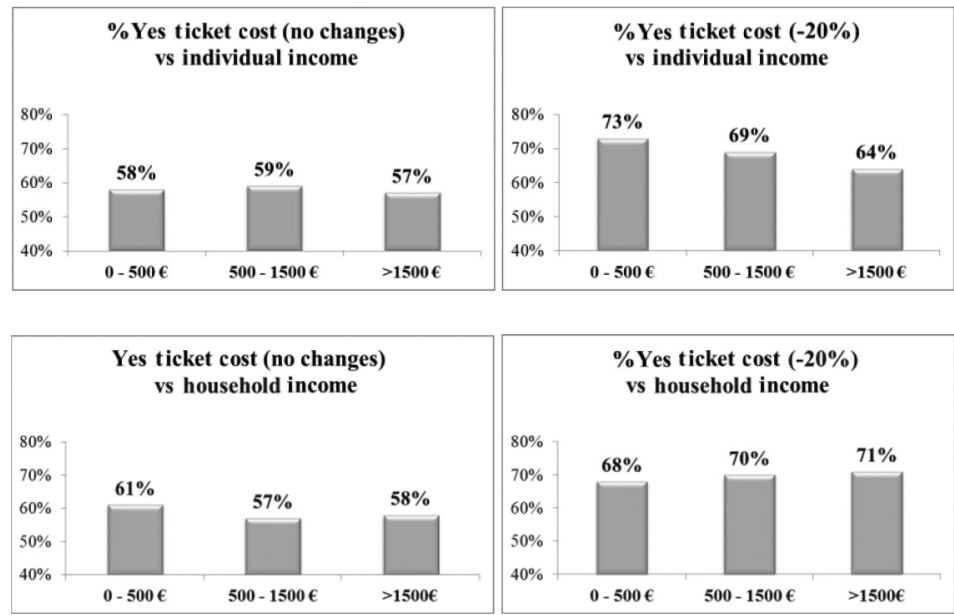

Figure 6: Yes percentages of ticket travel cost (actual situation and level '-20\%') vs individual and household income. 
ticket costs, since the latter probably represents the most important part in the travel cost. A medium-high sensitivity for the access/egress travel time is perceived. Moreover, it seems that there is a limited knowledge about HSR attributes and a low sensitivity for increasing travel costs of the transport mode chosen, which can suggest investments in HSR systems by reducing ticket costs and, even more important, access/egress travel times and costs, rather than a policy of car usage limitation by increasing costs.

Future researches consist in an application of a statistical analysis for discrete/classification variables, such as CATANOVA, to assess the conclusion above described and in specification and calibration of a mode choice model and the application of the methodology proposed in this paper to other case studies.

\section{REFERENCES}

[1] Lucas K., Making the connections between transport disadvantage and the social exclusion of low income populations in the Tshwane Region of South Africa. Journal of Transport Geography, 19, pp. 1320-1334, 2011. DOI: 10.1016/j.jtrangeo.2011.02.007

[2] Monzón, A, Ortega, E. \& López, E., Efficiency and spatial equity impacts of highspeed rail extensions in urban areas. Cities 30, pp. 18-30, 2013. DOI: 10.1016/ j.cities.2011.11.002

[3] Thomopoulos, N., Grant-Muller, S. \& Tight, M.R., Incorporating equity considerations in transport infrastructure evaluation: Current practice and a proposed methodology. Evaluation and Program Planning, 32, pp. 351-359, 1998.

[4] Tale, E., Visualizing fairness: Equity maps for planners. Journal of the American Planning Association, 1, pp. 22-38, 1998.

[5] Monzón, A., Ortega, E. \& López, E., Social impacts of high speed rail projects: addressing spatial equity effects. Proceedings of the 12th WCTR, 11-15 July, Lisbon, Portugal, 2010.

[6] D’Acierno, L., Gallo, M., Montella, B. \& Placido, A., The definition of a model framework for managing rail systems in the case of breakdowns. Proceedings of the 16th IEEE Conference on Intelligent Transportation Systems (ITSC), pp. 1059-1064, 2013.

[7] D’Acierno, L., Placido, A., Botte, M. \& Montella, B., A methodological approach for managing rail disruptions with different perspectives. International Journal of Mathematical Models and Methods in Applied Sciences, 10, pp. 80-86, 2016.

[8] Church, A., Frost, M. \& Sullivan, K., Transport and social exclusion in London. Transport Policy, 7, pp. 195-205, 2000. DOI: 10.1016/S0967-070X(00)00024-X

[9] Cascetta, E. \& Gentile, D., La 'Metropolitana d'Italia' per il rilancio del trasporto ferroviario. La ferrovia nelle aree metropolitane italiane, eds. M. De Luca, \& F. Pagliara, Aracne, Roma, pp. 11-37, 2007.

[10] Cascetta, E., Papola, A., Pagliara, F. \& Marzano, V., Analysis of mobility impacts of the high speed Rome-Naples rail link using within-day dynamic mode service choice models. Journal of Transport Geography, 19, pp. 635-643, 2011. DOI: 10.1016/ j.jtrangeo.2010.07.001

[11] Lucas K., Transport and social exclusion: Where are we now? Transport Policy, 20, pp. 105-113, 2012. DOI: 10.1016/j.tranpol.2012.01.013

[12] Lucas, K. \& Musso, A., Policies for social inclusion in transportation: An introduction to the special issue. Case Studies on Transport Policy, 2, pp. 37-40, 2014. DOI: 10.1016/ j.cstp.2014.06.002

[13] Masser, I., Sviden, O. \& Wegener, M., Transport planning for equity and sustainability. Transport Planning and Technology, 17, pp. 319-330, 1993. 